\title{
AN EXTENSION OF CARLITZ'S BIPARTITION IDENTITY
}

\author{
GEORGE E. ANDREWS ${ }^{1}$
}

ABSTRACT. Carlitz's bipartition identity is extended to a multipartite partition identity by the introduction of the summatory maximum function:

$$
\operatorname{smax}\left(n_{1}, n_{2}, \ldots, n_{r}\right)=n_{1}+n_{2}+\cdots+n_{r}-(r-1) \min \left(n_{1}, n_{2}, \ldots, n_{r}\right) .
$$

Let $\pi_{0}\left(n_{1}, n_{2}, \ldots, n_{r}\right)$ denote the number of partitions of $\left(n_{1}, n_{2}, \ldots, n_{r}\right)$ in which the minimum coordinate of each part is not less then the summatory maximum of the next part. Let $\pi_{1}\left(n_{1}, n_{2}, \ldots, n_{r}\right)$ denote the number of partitions of $\left(n_{1}, n_{2}, \ldots, n_{r}\right)$ in which each part has one of the $2 r-1$ forms: $(a+1, a, a, \ldots, a),(a, a+1, a, \ldots, a), \ldots,(a, a, a, \ldots, a+1),(r a+2, r a$ $+2, \ldots, r a+2), \quad(r a+3, r a+3, \ldots, r a+3), \ldots,(r a+r, r a+r, \ldots, r a$ $+r)$. Theorem: $\pi_{0}\left(n_{1}, \ldots, n_{r}\right)=\pi_{1}\left(n_{1}, \ldots, n_{r}\right)$.

1. Introduction. In 1963, L. Carlitz [3], [5] proved the following identity related to the partitions of bipartite numbers. Recall that a bipartite number is an ordered pair $(n, m)$ of nonnegative integers not both zero. We define $\pi(n, m)$ as the number of partitions of the bipartite $(n, m)$ of the form

$$
(n, m)=\left(n_{1}, m_{1}\right)+\left(n_{2}, m_{2}\right)+\cdots+\left(n_{r}, m_{r}\right)
$$

(addition is coordinate-wise) subject to the condition

$$
\min \left(n_{i}, m_{i}\right) \geqslant \max \left(n_{i+1}, m_{i+1}\right) .
$$

We define $\pi^{*}(n, m)$ as the number of partitions of $(n, m)$ in which each part is one of the forms $(a-1, a),(a, a-1)$, or $(2 a, 2 a)$.

CARLItz's Theorem. For every bipartite $(n, m): \pi(n, m)=\pi^{*}(n, m)$.

Apart from the intrinsic elegance of this theorem, there is also the interesting fact that this is the only bipartition identity that is not merely the consequence of elementary infinite product identities (such as the generalization of Euler's theorem given by M. S. Cheema [7] and the generalization of Euler pairs given by M. V. Subbarao [10]). Indeed Carlitz's theorem is more

Received by the editors April 12, 1976 and, in revised form, September 18, 1976.

AMS (MOS) subject classifications (1970). Primary 05A15, 05A17, 05A19, 10A45.

Key words and phrases. Partitions, multipartite numbers.

${ }^{1}$ Supported in part by the United States Army under Contract No. DAAG29-75-C-0024, the National Science Foundation Grant No. 23774 and the Deutsche Forschungs Gemeinschaft through the University of Erlangen. 
like the Rogers-Ramanujan identities [1, Chapter 14] in that one partition function $\pi(n, m)$ is concerned with a "difference condition" between the parts while the second $\pi^{*}(n, m)$ is concerned only with the form of each part individually.

In subsequent papers, L. Carlitz and D. P. Roselle [5], [6], [8], [9] studied an obvious generalization $\pi\left(n_{1}, \ldots, n_{r}\right)$ of $\pi(n, m)$ to multipartite numbers, namely $\pi\left(n_{1}, \ldots, n_{r}\right)$ is the number of partitions of the $r$-partite number $\left(n_{1}, \ldots, n_{r}\right)$ of the form

$$
\left(n_{1}, \ldots, n_{r}\right)=\left(m_{11}, \ldots, m_{1 r}\right)+\left(m_{21}, \ldots, m_{2 r}\right)+\cdots+\left(m_{s 1}, \ldots, m_{s r}\right)
$$

subject to the condition $\min \left(m_{j 1}, \ldots, m_{j r}\right) \geqslant \max \left(m_{j+1,1}, \ldots, m_{j+1, r}\right)$. While many interesting results were obtained for $\pi\left(n_{1}, \ldots, n_{r}\right)$ related to certain generalized Eulerian functions, no other identity like Carlitz's theorem was found.

In this paper we shall generalize Carlitz's theorem to $r$-partite numbers. To do this we define the summatory maximum "smax" of $\left(n_{1}, \ldots, n_{r}\right)$ by

$$
\operatorname{smax}\left(n_{1}, \ldots, n_{r}\right)=n_{1}+n_{2}+\cdots+n_{r}-(r-1) \min \left(n_{1}, \ldots, n_{r}\right)
$$

Note that $\operatorname{smax}\left(n_{1}, \ldots, n_{r}\right)=\max \left(n_{1}, \ldots, n_{r}\right)$ when $r=1$ or 2 .

Definition 1. Let $\pi_{0}\left(n_{1}, \ldots, n_{r}\right)$ denote the number of partitions of the $r$ partite number

$$
\left(n_{1}, \ldots, n_{r}\right)=\left(m_{11}, \ldots, m_{1 r}\right)+\cdots+\left(m_{s 1}, \ldots, m_{s r}\right)
$$

subject to the condition: $\min \left(m_{j 1}, \ldots, m_{j r}\right) \geqslant \operatorname{smax}\left(m_{j+1,1}, \ldots, m_{j+1, r}\right)$.

Definition 2. Let $\pi_{1}\left(n_{1}, \ldots, n_{r}\right)$ denote the number of partitions of $\left(n_{1}, \ldots, n_{r}\right)$ in which each part has one of the $2 r-1$ possible forms: $(a+1, a, a, \ldots, a),(a, a+1, a, \ldots, a), \ldots,(a, a, \ldots, a+1),(r a+2, r a+2$, $\ldots, r a+2),(r a+3, r a+3, \ldots, r a+3), \ldots,(r a+r, r a+r, \ldots, r a$ $+r)($ for $a \geqslant 0)$.

THEOREM 1. For every r-partite number $\left(n_{1}, \ldots, n_{r}\right)$,

$$
\pi_{0}\left(n_{1}, \ldots, n_{r}\right)=\pi_{1}\left(n_{1}, \ldots, n_{r}\right)
$$

We remark that this theorem is trivial when $r=1$ in that $\pi_{0}(n)=\pi_{1}(n)$ $=p(n)$, the number of partitions of $n$. When $r=2$, Theorem 1 reduces to Carlitz's theorem since $\operatorname{smax}\left(n_{1}, n_{2}\right)=\max \left(n_{1}, n_{2}\right)$.

In $\$ 2$ we shall prove Theorem $1 ; \S 3$ concludes with a brief discussion of open questions and a closed form expression for

$$
\sum_{n_{1}, \ldots, n_{r} \geqslant 0} \operatorname{smax}\left(n_{1}, n_{2}, \ldots, n_{r}\right) x_{1}^{n_{1}} x_{2}^{n_{2}} \cdots x_{r}^{n_{r}}
$$

Carlitz [2] has treated a similar problem when $\operatorname{smax}\left(n_{1}, n_{2}, \ldots, n_{r}\right)$ is replaced by $\max \left(n_{1}, n_{2}, \ldots, n_{r}\right)$ or $\min \left(n_{1}, n_{2}, \ldots, n_{r}\right)$. 
2. Proof of Theorem 1. Our proof is quite similar to that given by Carlitz for bipartite numbers. We begin by noting the algebraic identity

$$
\sum_{j=1}^{r}(-1)^{j+1} \sum_{1 \leqslant i_{1}<\cdots<i_{j} \leqslant r} \mathbb{Q}_{i_{1}} \cdots \mathbb{Q}_{i_{j}}=1-\prod_{i=1}^{r}\left(1-\mathbb{Q}_{i}\right)
$$

which we need later in the proof.

We now define $\pi\left(n_{1}, \ldots, n_{r} \mid a_{1}, \ldots, a_{r}\right)$ as the number of partitions (like (1.2)) of $\left(n_{1}, \ldots, n_{r}\right)$ subject to the conditions of Definition 1 with the added restriction that $\min \left(a_{1}, \ldots, a_{r}\right) \geqslant \operatorname{smax}\left(m_{11}, \ldots, m_{1 r}\right)$.

$$
\xi_{a_{1} a_{2} \cdots a_{r}}=\sum_{n_{1}, \ldots, n_{r} \geqslant 0} \pi\left(n_{1}, \ldots, n_{r} \mid a_{1}, \ldots, a_{r}\right) x_{1}^{n_{1}} x_{2}^{n_{2}} \cdots x_{r}^{n_{r}} .
$$

By classifying the $r$-partite partitions admissible under Definition 1 according to their largest part, we immediately deduce

$$
\xi_{a_{1} a_{2} \cdots a_{r}}=\sum_{\substack{\operatorname{smax}\left(n_{1}, \ldots, n_{r}\right) \leqslant \min \left(a_{1}, \ldots, a_{r}\right) \\ n_{1}, \ldots, n_{r} \geqslant 0}} x_{1}^{n_{1}} x_{2}^{n_{2}} \cdots x_{r}^{n_{r}} \xi_{n_{1} n_{2}} \cdots n_{r} .
$$

Furthermore we see from the definition of $\pi\left(n_{1}, \ldots, n_{r} \mid a_{1}, \ldots, a_{r}\right)$, that if $a=\min \left(a_{1}, a_{2}, \ldots, a_{r}\right)$, then

$$
\xi_{a_{1} a_{2}} \cdots a_{r}=\xi_{a}
$$

where we have written $\xi_{a}=\xi_{a a} \ldots a$.

Next we let

$$
F(u)=\sum_{n=0}^{\infty} u^{n} \xi_{n}
$$

Hence

$$
\begin{aligned}
& F(u)=\sum_{n=0}^{\infty} u^{n} \sum_{\operatorname{smax}\left(n_{1}, \ldots, n_{r}\right) \leqslant n} x_{1}^{n_{1}} \cdots x_{r}^{n_{r}} \xi_{n_{1}} \cdots n_{r} \\
& =\sum_{n_{1} \geqslant 0, \ldots, n_{r} \geqslant 0} x_{1}^{n_{1}} \cdots x_{r}^{n_{r}} \xi_{n_{1}} \cdots n_{r} \sum_{n \geqslant \operatorname{smax}\left(n_{1}, \ldots, n_{r}\right)} u^{n} \\
& =\sum_{j=1}^{r}(-1)^{j+1} \sum_{1 \leqslant i_{1}<\cdots<i_{j} \leqslant r} \sum_{\substack{\min \left(n_{1}, \ldots, n_{r}\right) \\
=n_{i_{1}}=\cdots=n_{i_{r}}}} x_{1}^{n_{1}} \cdots x_{r}^{n_{r}} \xi_{n_{i_{1}}} \\
& \sum_{n \geqslant n_{1}+\cdots+n_{r}-(r-1) n_{i_{1}}} u^{n} \\
& =(1-u)^{-1} \sum_{j=1}^{r}(-1)^{j+1} \sum_{1 \leqslant i_{1}<\cdots<i_{j} \leqslant r} \sum_{\substack{\min \left(n_{1}, \ldots, n_{r}\right) \\
=n_{i_{1}}=\cdots=n_{i_{r}}}}\left(u x_{1}\right)^{n_{1}} \\
& \cdots\left(u x_{r}\right)^{n_{r}} u^{-(r-1) n_{i_{1}}} \xi_{n_{i_{1}}}
\end{aligned}
$$




$$
\begin{aligned}
& =(1-u)^{-1} \sum_{j=1}^{r}(-1)^{j+1} \sum_{1 \leqslant i_{1}<\cdots<i_{j} \leqslant r} \prod_{i \neq i_{1}, \ldots, i_{j}}\left(1-u x_{i}\right)^{-1} \sum_{n_{i_{1}}=0}^{\infty}\left(x_{1} \cdots x_{r} u\right)^{n_{i_{1}} \xi_{n_{i_{1}}}} \\
& =(1-u)^{-1} F\left(x_{1} \cdots x_{r} u\right) \sum_{j=1}^{r}(-1)^{j+1} \sum_{1 \leqslant i_{1}<\cdots<i_{j} \leqslant r} \prod_{i \neq i_{1}, \ldots, i_{j}}\left(1-u x_{i}\right)^{-1} \\
& =\frac{F\left(x_{1} \cdots x_{r} u\right)}{(1-u)\left(1-u x_{1}\right) \cdots\left(1-u x_{r}\right)} \sum_{j=1}^{r}(-1)^{j+1} \sum_{1 \leqslant i_{1}<\cdots<i_{j} \leqslant r}\left(1-u x_{i_{1}}\right) \cdots\left(1-u x_{i_{r}}\right) \\
& =\frac{F\left(x_{1} \cdots x_{r} u\right)\left(1-x_{1} \cdots x_{r} u^{r}\right)}{(1-u)\left(1-u x_{1}\right) \cdots\left(1-u x_{r}\right)},
\end{aligned}
$$

where we have used (2.1) with $\mathbb{Q}_{k}=1-u x_{k}$. Iteration of (2.6) yields

$$
F(u)=\frac{\prod_{n=0}^{\infty}\left(1-x_{1}^{r n+1} x_{2}^{r n+1} \cdots x_{r}^{r n+1} u^{r}\right)}{\prod_{n=0}^{\infty}\left(1-u x_{1}^{n} \cdots x_{r}^{n}\right)\left(1-u x_{1}^{n+1} x_{2}^{n} \cdots x_{r}^{n}\right) \cdots\left(1-u x_{1}^{n} x_{2}^{n} \cdots x_{r}^{n+1}\right)} .
$$

Finally,

$$
\begin{aligned}
& \sum_{n_{1}, \ldots, n_{r} \geqslant 0} \pi_{0}\left(n_{1}, \ldots, n_{r}\right) x_{1}^{n_{1}} \cdots x_{r}^{n_{r}} \\
= & \lim _{n \rightarrow \infty} \xi_{n}=\lim _{u \rightarrow 1^{-}}(1-u) F(u) \quad \text { (by Abel's Lemma) } \\
= & \frac{1}{\prod_{\substack{n=1 \\
n \neq 1(\bmod r)}}^{\infty}\left(1-x_{1}^{n} x_{2}^{n} \cdots x_{r}^{n}\right) \prod_{m=0}^{\infty}\left(1-x_{1}^{m+1} x_{2}^{m} \cdots x_{r}^{m}\right) \cdots\left(1-x_{1}^{m} x_{2}^{m} \cdots x_{r}^{m+1}\right)} \\
= & \sum_{n_{1}, \ldots, n_{r} \geqslant 0} \pi_{1}\left(n_{1}, \ldots, n_{r}\right) x_{1}^{n_{1}} x_{2}^{n_{2}} \cdots x_{r}^{n_{r}} .
\end{aligned}
$$

Theorem 1 now follows from a comparison of the coefficients of $x_{1}^{n_{1}} x_{2}^{n_{2}} \cdots x_{r}^{n_{r}}$ in equation (2.8).

3. Conclusion. It is natural to ask whether any other partition identities like Theorem 1 exist for multipartite numbers. The technique used here (as pointed out by Carlitz) arose in T. W. Chaundy's work on plane partitions. It is quite conceivable that elementary algebraic identities other than (2.1) might lead to further nice multipartite partition identities through Chaundy's method.

Finally we mention that Carlitz [2] has obtained closed form expressions for the $r$-variable generating functions of $\min \left(n_{1}, \ldots, n_{r}\right)$ and $\max \left(n_{1}, \ldots, n_{r}\right)$. In particular he proved that 


$$
\begin{aligned}
\sum_{n_{1}, \ldots, n_{r}} \geqslant 0 & \min \left(n_{1}, \ldots, n_{r}\right) x_{1}^{n_{1}} x_{2}^{n_{2}} \cdots x_{r}^{n_{r}} \\
& =\frac{x_{1} x_{2} \cdots x_{r}}{\left(1-x_{1}\right) \cdots\left(1-x_{r}\right)\left(1-x_{1} x_{2} \cdots x_{r}\right)} .
\end{aligned}
$$

From equation $(3.1)$ : we obtain immediately, the generating, function for $\operatorname{smax}\left(n_{1}, \ldots, n_{r}\right)$ :

$$
\begin{aligned}
& \sum_{n_{1}, \ldots, n_{r} \geqslant 0} \operatorname{smax}\left(n_{1}, \ldots, n_{r}\right) x_{1}^{n_{1}} x_{2}^{n_{2}} \cdots x_{r}^{n_{r}} \\
= & \sum_{n_{1}, \ldots, n_{r} \geqslant 0}\left(n_{1}+n_{2}+\cdots+n_{r}-(r-1) \min \left(n_{1}, n_{2}, \ldots, n_{r}\right)\right) x_{1}^{n_{1}} x_{2}^{n_{2}} \cdots x_{r}^{n_{r}} \\
= & \frac{x_{1}}{\left(1-x_{1}\right)^{2}\left(1-x_{2}\right) \cdots\left(1-x_{r}\right)}+\frac{x_{2}}{\left(1-x_{1}\right)\left(1-x_{2}\right)^{2}\left(1-x_{3}\right) \cdots\left(1-x_{r}\right)} \\
& +\cdots+\frac{x_{r}}{\left(1-x_{1}\right)\left(1-x_{2}\right) \cdots\left(1-x_{r-1}\right)\left(1-x_{r}\right)^{2}} \\
& -\frac{(r-1) x_{1} x_{2} \cdots x_{r}}{\left(1-x_{1}\right)\left(1-x_{2}\right) \cdots\left(1-x_{r}\right)\left(1-x_{1} x_{2} \cdots x_{r}\right)} \\
= & \left(1-x_{1}\right)^{-1}\left(1-x_{2}\right)^{-1} \cdots\left(1-x_{r}\right)^{-1}\left\{\sum_{j=1}^{r} \frac{x_{j}}{1-x_{j}}-\frac{(r-1) x_{1} x_{2} \cdots x_{r}}{1-x_{1} x_{2} \cdots x_{r}}\right\} .
\end{aligned}
$$

I wish to thank Professor Klaus Leeb and his colleagues at Erlangen for their support and interest during the writing of this paper.

\section{REFERENCES}

1. G. E. Andrews, Number theory, Saunders, Philadelphia, 1971. MR 46 \#8943.

2. L. Carlitz, The generating function for $\max \left(n_{1}, \ldots, n_{k}\right)$, Portugal. Math. 21 (1962), 201-207. MR 29 \#5753.

3. - Some generating functions, Duke Math. J. 30 (1963), 191-201. MR 26 \#6063.

4. —_ A problem in partitions, Duke Math. J. 30 (1963), 203-213. MR 26 \#6143.

5. - Generating functions and partitions problems, Proc. Sympos. Pure Math., vol. 8, Amer. Math. Soc., Providence, R.I., 1965, pp. 144-169. MR 31 \# 72.

6. L. Carlitz and D. P. Roselle, Restricted bipartite partitions, Pacific J. Math. 19 (1966), 221-228. MR 34 \#2549.

7. M. S. Cheema, Vector partitions and combinatorial identities, Math. Comp. 18 (1964), 414-420. MR 29 \# 4697.

8. D. P. Roselle, Generalized Eulerian functions and a problem in partitions, Duke Math. J. 33 (1966), 293-304. MR 32 \#7532.

9. - Restricted k-partite partitions, Math. Nachr. 32 (1966), 139-148. MR 34 \#2550.

10. M. V. Subbarao, Partition theorems for Euler pairs, Proc. Amer. Math. Soc. 28 (1971), 330-336. MR 43 \#175.

Department of Mathematics, Pennsylvania State University, University Park, PennsylVANIA 16802 (Current address)

Department of Mathematics, University of Erlangen, Erlangen, Grrmany

Department of Mathematics, University of Wisconsin, Madison, Wisconsin 53706 